\title{
Cisplatin-based chemoradiotherapy with 5-fluorouracil or pemetrexed in patients with locally advanced, unresectable esophageal squamous cell carcinoma: A retrospective analysis
}

\author{
ZENGYUN LI ${ }^{1}$, PEILIANG ZHANG ${ }^{2}$, QINGTONG MA ${ }^{1}$, DONGQING WANG ${ }^{3}$ and TAO ZHOU ${ }^{3}$ \\ ${ }^{1}$ Department of Oncology, Laiwu Municipal Hospital of Traditional Chinese Medicine, Laiwu, \\ Shandong 271100; ${ }^{2}$ Department of Radiation Oncology, Yishui Central Hospital, Yishui, Shandong 276400; \\ ${ }^{3}$ Department of Radiation Oncology, Shandong Tumor Hospital, Jinan, Shandong 250117, P.R. China
}

Received March 10, 2016; Accepted October 20, 2016

DOI: $10.3892 / \operatorname{mco} .2017 .1222$

\begin{abstract}
Treatment with 5-fluorouracil (5-FU) and cisplatin (PF regimen) remains the most frequently used chemotherapy for esophageal squamous cell carcinoma (SCC). The aim of the present study was to assess the efficacy and safety of pemetrexed/cisplatin (PP regimen) as definitive treatment compared with PF. A total of 60 patients with locally advanced, unresectable SCC of the esophagus receiving concomitant chemoradiotherapy were recruited in this study; of those patients, 29 received four cycles (two concomitant and two post-radiotherapy) of the PF regimen (arm A, cisplatin $25 \mathrm{mg} / \mathrm{m}^{2} /$ day i.v. on days $1-3$ plus 5 -FU $800 \mathrm{mg} / \mathrm{m}^{2} / 24 \mathrm{~h}$ by continuous infusion on days 1-5) and 31 received four cycles of the PP regimen ( $\mathrm{arm} \mathrm{B}$, cisplatin $25 \mathrm{mg} / \mathrm{m}^{2} /$ day i.v. on days 1-3 plus pemetrexed $500 \mathrm{mg} / \mathrm{m}^{2}$ on day 1). All the patients in both arms received a total radiation dose of $59.6 \mathrm{~Gy}$. The two arms were well-matched for age, gender, Karnofsky performance status, TNM stage, tumor location and length. The overall response rate was $89.7 \%$ in arm A vs. $93.5 \%$ in arm $\mathrm{B}(\mathrm{P}>0.05)$. The median overall survival was 26.1 months [95\% confidence interval (CI): 15.3-36.8 months] in arm A vs. 28.7 months (95\% CI: 9.4-48.0 months) in arm B ( $\mathrm{P}>0.05)$. Severe esophagitis occurred in $31.0 \%$ (9/29) of the patients in arm A vs. $12.9 \%$ (4/31) of the patients in arm B; the difference was statistically significant $(\mathrm{P}=0.036)$. Grade $3 / 4$ leukopenia and thrombocytopenia occurred in $4(13.8 \%)$ and $1(3.4 \%)$ patients, respectively, in arm A vs. $12(38.7 \%)$ and $6(19.4 \%)$ patients, respectively, in arm B; the differences were statistically significant $(\mathrm{P}=0.029$ and 0.041 , respectively). Therefore, chemoradiotherapy with the $\mathrm{PP}$ regimen achieved therapeutic
\end{abstract}

Correspondence to: Dr Dongqing Wang, Department of Radiation Oncology, Shandong Tumor Hospital, 440 Jiyan Road, Jinan, Shandong 250117, P.R. China

E-mail: wdqlove2009@sina.com

Key words: esophageal, squamous cell carcinoma, chemoradiotherapy, pemetrexed, 5-fluorouracil results comparable with those of the PF regimen; in terms of toxicity, the incidence of hematological toxicity was higher and that of esophagitis was lower with the PP regimen.

\section{Introduction}

Concurrent chemoradiotherapy has been established as one of the standard therapies for patients with locally advanced, unresectable esophageal carcinoma based on the results of the Radiation Therapy Oncology Group (RTOG) 85-01 and 95-04 trials, which demonstrated a significant survival advantage of concurrent chemoradiation over radiation alone $(1,2)$. However, a standard and effective chemotherapeutic regimen for combining with radiotherapy has not yet been established.

Although the standard chemotherapeutic agents for esophageal carcinoma have not yet been determined, various types of chemotherapy regimens have been investigated in an attempt to prolong survival and improve quality of life. The most frequently used chemotherapeutic agents in esophageal cancer treatment are combined cisplatin and 5-fluorouracil (5-FU) (1-3). A phase II study (3) by the Japan Clinical Oncology Group reported that the complete response rate with cisplatin/5-FU (PF regimen) and radiotherapy for stage II-III esophageal squamous cell carcinoma (SCC) achieved a response rate of $62.2 \%$ (46/74); the median survival time was 29 months, with 3- and 5-year survival rates of 44.7 and $36.8 \%$, respectively. However, half the cases in these series of patients included potentially resectable carcinomas. A better prognosis with chemoradiotherapy in esophageal SCC was reported by Zhao et al (4), with a median survival time of 30.8 months and a 5-year survival rate of $40 \%$ for stage I-III patients treated with the PF regimen combined with late-course accelerated hyperfractionated radiotherapy (LCAHRT).

In order to increase the therapeutic ratio over that of standard PF-based chemoradiotherapy, attempts have been made in a phase I/II study to incorporate next-generation cytotoxic chemotherapeutic agents, such as docetaxel $(5,6)$. However, survival remains disappointing and did not improve with the standard PF regimen. Treatment-related toxicities may compromise clinical efficacy (6). Therefore, new drugs and combinations with a better therapeutic index are required. 
More recently, pemetrexed was introduced in phase I trials for esophageal SCC and the preliminary results are promising (7). As a novel antimetabolite, pemetrexed acts as a multitargeted antifolate by inhibiting several key enzymes involved in nucleotide synthesis (8). Pemetrexed, as a single agent or combined with platinum, has also demonstrated broad antitumor activity in a wide variety of solid tumors (9). In a phase I trial (7), pemetrexed was evaluated in combination with cisplatin and concurrent selective lymph node LCAHRT for patients with locally advanced esophageal SCC; that study demonstrated that the maximum tolerated dose of pemetrexed was $500 \mathrm{mg} / \mathrm{m}^{2}$ and the recommended dose was $400 \mathrm{mg} / \mathrm{m}^{2}$. Although toxicities were common, the protocol was overall safe, well-tolerated, and achieved an encouraging outcome. One phase II study (10) investigated $500 \mathrm{mg} / \mathrm{m}^{2}$ neoadjuvant pemetrexed and carboplatin in conjunction with concomitant radiation of 50.4 Gy followed by surgery for locally advanced esophageal cancer and gastroesophageal junction tumors. This phase II study reported a $23 \%(6 / 26)$ pathological complete response and 22 patients underwent complete cancer resection, with a median survival time of 17.8 months [95\% confidence interval (CI): 12.2-30.7 months]. However, 22 patients had at least one grade $\geq 3$ adverse event, and 3 deaths were reported postoperatively.

To the best of our knowledge, until recently there were no published studies focusing on the efficacy and safety of pemetrexed/cisplatin (PP regimen) compared with the PF regimen in concomitant chemoradiotherapy. Therefore, the objective of the present study was to evaluate the combination of pemetrexed and cisplatin in patients with locally advanced, unresectable esophageal SCC.

\section{Patients and methods}

Design. A retrospective study was conducted to determine the efficacy and safety of the PP vs. the PF regimen in patients with locally advanced, unresectable esophageal SCC treated with concomitant chemoradiotherapy. The primary objective was to assess tumor response and overall survival, and the secondary objective was to assess treatment-related toxicity. The Institutional Review Board of the Shandong Tumor Hospital (Jinan, China) approved the protocol of this retrospective study and all the patients provided written informed consent.

Patient population and eligibility. Between January, 2004 and November, 2011, 72 eligible patients underwent concomitant LCAHRT and cisplatin-based chemotherapy with a curative intent for the treatment of locally advanced, unresectable esophageal SCC at the Shandong Tumor Hospital. The eligibility criteria for this study were as follows: i) Karnofsky performance status score $\geq 70$; ii) patients aged $\leq 75$ years; iii) histologically confirmed SCC, previously untreated; and iv) clinical stage T1-T4, N0/1, M0/1a according to the American Joint Committee staging system (2002) (11). The exclusion criteria included distant organ metastases, evidence of esophageal perforation and other serious underlying medical conditions.

Treatment evaluation and details. The pretreatment evaluation generally included complete history and physical examination, complete blood cell count and serum chemistry profile, endoscopy with biopsy, upper gastrointestinal, chest and abdominal computed tomography (CT) scans and bone scan with single photon emission CT. In order to exclude patients with distant organ metastases, a magnetic resonance imaging scan of the brain and neck and a whole-body ${ }^{18} \mathrm{~F}$-fluorodeoxyglucose positron emission tomography scan were performed as part of routine evaluation.

The treatment scheme is summarized in Fig. 1. All the patients were scheduled to receive two cycles of concurrent cisplatin-based chemotherapy and radiation [LCAHRT; $59.6 \mathrm{~Gy} / 34$ fractions (fx)], followed by an additional two cycles of consolidation chemotherapy.

Chemotherapy. Patients in arm A were treated with the PF regimen (intravenous infusion of cisplatin $25 \mathrm{mg} / \mathrm{m}^{2} /$ day on days 1-3 and continuous intravenous infusion of 5-fluorouracil $800 \mathrm{mg} / \mathrm{m}^{2} / 24 \mathrm{~h}$ on days $1-5$, every 21 days). Patients in arm $\mathrm{B}$ were treated with the PP regimen (intravenous infusion of cisplatin $25 \mathrm{mg} / \mathrm{m}^{2} /$ day on days $1-3$ and pemetrexed $500 \mathrm{mg} / \mathrm{m}^{2}$ on day 1 , every 21 days). All the patients treated with the PP regimen received folic acid, vitamin $\mathrm{B} 12$ and steroid prophylaxis. Appropriate antiemetics were prescribed, and human granulocyte colony-stimulating factor was permitted during treatment.

Radiotherapy. The radiation dose was the same in both arms. The radiation was delivered by 6-MV X-rays using a two-course irradiation schedule: The first course of radiation covered the primary tumors, metastatic regional lymph node(s) and high-risk nodal regions $(7,12)$, administered at 2 Gy per $\mathrm{fx}, 5 \mathrm{fx} /$ week, to a total dose of $40 \mathrm{~Gy}$ in $20 \mathrm{fx}$; the second course of radiation was delivered to the boost volume for an additional dose of 19.6 Gy twice a day in $14 \mathrm{fx}$ within 7 days at $1.4 \mathrm{~Gy} / \mathrm{fx}$, with a 6-h minimal interval between fractions. The total dose administered to the clinical tumor was $59.6 \mathrm{~Gy} / 34$ fx/35 days.

Treatment assessments. Tumor response was assessed according to the Response Evaluation Criteria in Solid Tumors (RECIST) guidelines (13). The response criteria for the target lesions are as follows: Complete response (CR), disappearance of all target lesions; partial response (PR), $\geq 30 \%$ decrease; stable disease (SD), neither PR nor PD criteria met; and progressive disease (PD), $\geq 20 \%$ increase or appearance of new lesion(s). The overall survival was calculated from the date of radiotherapy initiation until death or the last follow-up evaluation.

Treatment-related toxicity and follow-up. Treatment-related toxicity assessment was performed at least weekly during treatment, 4 weeks after completion of therapy, every 3 months for 2 years and every 6 months thereafter, using the National Cancer Institute Common Toxicity Criteria, version 3.0 (14). A full history and physical examination, as well as repeat blood work, were conducted at these visits. Spiral CT scans of the chest and upper gastrointestinal tract were obtained at every follow-up examination to evaluate the status of the disease.

Statistical analysis. The statistical analysis was performed using SPSS software, version 10.0 (SPSS Inc., Chicago, IL, USA). The survival analysis was performed using the 
Table I. Patient baseline characteristics

\begin{tabular}{lccc}
\hline Characteristics & $\begin{array}{c}\text { PF } \\
(\text { arm A; } \mathrm{n}=29)\end{array}$ & $\begin{array}{c}\text { PP } \\
\text { (arm B; } \mathrm{n}=31)\end{array}$ & P-value \\
\hline $\begin{array}{l}\text { Age (years) } \\
\text { Median (range) }\end{array}$ & $62(42-75)$ & $62(40-75)$ & 0.722 \\
$\begin{array}{l}\text { Gender } \\
\text { Male:female }\end{array}$ & $24: 5$ & $28: 3$ & 0.389 \\
$\begin{array}{l}\text { Karnofsky performance status } \\
\text { Median (range) }\end{array}$ & $80(70-100)$ & $80(70-100)$ & 0.898 \\
$\begin{array}{l}\text { Location } \\
\text { Cervical:upper:middle:lower }\end{array}$ & $4: 11: 12: 2$ & $3: 12: 12: 4$ \\
$\begin{array}{l}\text { Stage } \\
\text { IIa:IIb:III:IVa }\end{array}$ & $4: 0: 16: 9$ & $6: 3: 12: 10$ & 0.787 \\
$\begin{array}{l}\text { Tumor length (cm) } \\
\text { Median (range) }\end{array}$ & $6.5(2.0-11.5)$ & $5.0(3.5-10.0)$ & 0.576 \\
\hline
\end{tabular}

PF, cisplatin and 5-fluorouracil; PP, cisplatin and pemetrexed.

Table II. Treatment response according to RECIST.

\begin{tabular}{lccc}
\hline $\begin{array}{l}\text { Type of } \\
\text { response, } \\
\mathrm{n}(\%)\end{array}$ & $\begin{array}{c}\mathrm{PF} \\
(\mathrm{arm} \mathrm{A} ; \\
\mathrm{n}=29)\end{array}$ & $\begin{array}{c}\mathrm{PP} \\
(\operatorname{arm~B} ; \\
\mathrm{n}=31)\end{array}$ & $\begin{array}{c}\text { Overall } \\
(\mathrm{n}=60)\end{array}$ \\
\hline $\mathrm{CR}$ & $16(55.2)$ & $13(41.9)$ & $29(48.3 \%)$ \\
$\mathrm{PR}$ & $10(34.5)$ & $16(51.6)$ & $26(43.3 \%)$ \\
$\mathrm{RR}(\mathrm{CR}+\mathrm{PR})$ & $26(89.7)$ & $29(93.5)$ & $55(91.7 \%)$ \\
$\mathrm{SD}$ & $3(10.3)$ & $1(3.2)$ & $4(6.7 \%)$ \\
$\mathrm{PD}$ & $0(0.0)$ & $1(3.2)$ & $1(1.7 \%)$
\end{tabular}

RECIST, Response Evaluation Criteria In Solid Tumors; PF, cisplatin and 5-fluorouracil; PP, cisplatin and pemetrexed; CR, complete response; $\mathrm{PR}$, partial response; $\mathrm{SD}$, stable disease; $\mathrm{PD}$, progressive disease; RR, response rate.

actuarial Kaplan-Meier method, and differences between the curves were analyzed using the log-rank test. The constituent ratio was analyzed using the Chi-squared test. All statistical comparisons were performed with two-tailed tests on an intent-to-treat basis. A P-value of $<0.05$ was considered statistically significant.

\section{Results}

Patient characteristics. Between January, 2004 and November, 2011, 29 patients were treated with the PF regimen (arm A) and 31 patients with the PP regimen (arm B). All the patients completed the treatment schedules and were assessable for treatment efficacy and toxicity.

The two arms were similar for baseline characteristics (Table I). Although more patients with early-stage disease (IIa+IIb) were included in arm B (29.0\%) compared with arm A (13.8\%), the difference was not statistically significant

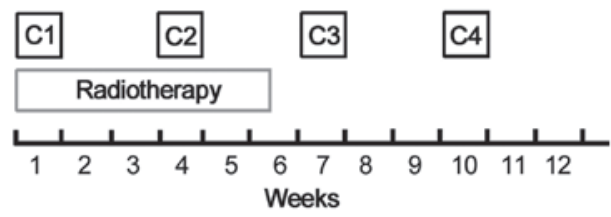

Figure 1. Treatment schedule. C, cycle.

$(\mathrm{P}=0.266)$. The majority of the patients had stage III and IVa disease located in the thoracic esophagus.

Treatment response. According to RECIST, a response was reported in 26 of the 29 patients $(89.7 \%)$ in arm A and in 29 of the 31 patients $(93.5 \%)$ in arm B (Table II). The difference was not statistically significant $(\mathrm{P}=0.304)$.

Survival. The median follow-up was 25.1 months in both arms. The median survival time was 26.1 months (95\% CI: 15.3-36.8 months) in arm A and 28.7 months (95\% CI: 9.4-48.0 months) in arm B. At 1, 3 and 5 years, the overall survival rate was $84.4,42.2$ and $33.2 \%$ in arm A, and 71.3, 51.9 and $40.9 \%$ in arm B. However, there were no significant difference between arm $\mathrm{A}$ and arm $\mathrm{B}$ in terms of long-term survival (Chi-squared=0.034, $\mathrm{P}=0.853$ ) (Fig. 2).

Toxicity. As demonstrated in Table III, the most frequently reported severe (grade $\geq 3$ ) adverse effects were hematological toxicity and esophagitis. Treatment-related severe leukopenia and thrombocytopenia occurred in $4(13.8 \%)$ and $1(3.4 \%)$ patients, respectively, in arm A, vs. 12 (38.7\%) and $6(19.4 \%)$ patients, respectively, in arm B; the differences were statistically significant ( $\mathrm{P}=0.029$ and 0.041 , respectively). Severe anaemia was reported in $3.4 \%$ ( 1 patient developed grade 4 anaemia) of the patients in arm A, vs. $12.9 \%$ in arm B; however, the difference was not statistically significant $(\mathrm{P}=0.059)$. A total of 9 patients $(31.0 \%)$ in arm A and 4 patients (12.9\%) in arm 
Table III. Treatment-related hematological and non-hematological toxicity.

\begin{tabular}{|c|c|c|c|}
\hline Toxicity & $\begin{array}{l}\text { PF (arm } \\
A ; n=29)\end{array}$ & $\begin{array}{l}\mathrm{PP}(\operatorname{arm} \\
\mathrm{B} ; \mathrm{n}=31)\end{array}$ & $\mathrm{P}$-value \\
\hline Leukopenia & & & 0.029 \\
\hline Grade $0-1: 2: 3: 4: 5$ & $12: 13: 4: 0: 0$ & $4: 15: 11: 1: 0$ & \\
\hline Anaemia & & & 0.059 \\
\hline Grade $0-1: 2: 3: 4: 5$ & $28: 0: 0: 1: 0$ & $25: 2: 4: 0: 0$ & \\
\hline Thrombocytopenia & & & 0.041 \\
\hline Grade $0-1: 2: 3: 4: 5$ & $23: 5: 1: 0: 0$ & $19: 6: 4: 2: 0$ & \\
\hline Vomiting & & & 0.051 \\
\hline Grade $0-1: 2: 3: 4: 5$ & $25: 4: 0: 0: 0$ & 20:10:1:0:0 & \\
\hline Pneumonitis & & & 0.170 \\
\hline Grade $0-1: 2: 3: 4: 5$ & $29: 0: 0: 0: 0$ & $30: 1: 0: 0: 0$ & \\
\hline Esophagitis & & & 0.036 \\
\hline Grade $0-1: 2: 3: 4: 5$ & $2: 18: 9: 0: 0$ & $3: 24: 4: 0: 0$ & \\
\hline
\end{tabular}

Bold print indicates statistical significance. PF, cisplatin and 5-fluorouracil; PP, cisplatin and pemetrexed.

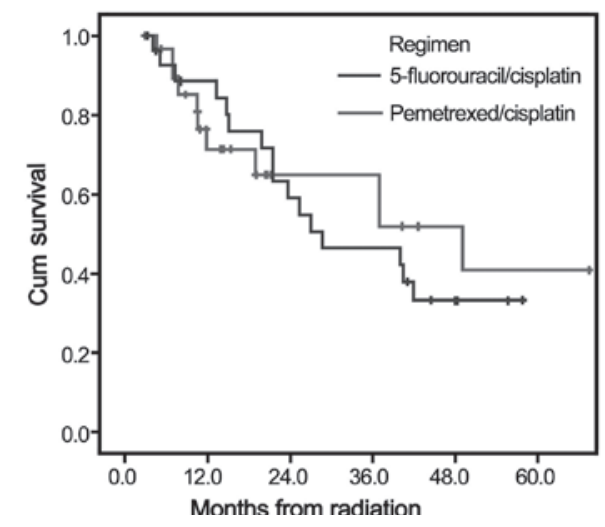

Figure 2. Kaplan-Meier curve of overall survival for patients treated with 5-fluorouracil /cisplatin (PF regimen) vs. pemetrexed/cisplatin (PP regimen). Cum, cumulative.

$\mathrm{B}$ developed grade 3 radiation esophagitis and the difference was statistically significant $(\mathrm{P}=0.036)$. Severe gastrointestinal reactions were infrequent in both arms. No patients developed grade $\geq 3$ radiation pneumonitis.

Six deaths were considered to be possibly related to the treatment regimens: 4 in arm A (upper gastrointestinal hemorrhage occurred in 1 patient and esophagotracheal fistula in 3 patients), and 2 in arm B (1 case each of upper gastrointestinal hemorrhage and esophagotracheal fistula); all patients were male and had stage T4 disease.

\section{Discussion}

On the basis of previous clinical phase I study, $400 \mathrm{mg} / \mathrm{m}^{2}$ pemetrexed administered on day 1 once every 21 days was the recommended regimen for locally advanced esophageal SCC (7) in combination with cisplatin-based chemoradiotherapy. Although limited by the small number of patients, our data suggest that the administration of pemetrexed may be feasible and well-tolerated in combination with radiotherapy; furthermore, this PP-based chemoradiotherapy achieved a tumor response rate of $93.5 \%$, with acceptable toxicity and only 2 possibly treatment-related deaths. The control arm with the PF regimen exhibited a mildly inferior response rate $(89.7 \%)$, whereas there were 4 reported toxicity-related deaths. The present study demonstrated that the incidence of hematological toxicities was higher with the PP compared with the PF regimen, which should be taken into consideration. However, the incidence of esophagitis with the $\mathrm{PP}$ regimen was lower compared with that with the PF regimen. The median survival in the PF arm was 26.1 months (95\% CI: 5.3-36.8 months) and was superior to those reported by the RTOG 85-01 and RTOG 94-05 trials (14.1 and 18.1 months, respectively) $(1,2)$. With the PP regimen, the median survival in our trial was 28.7 months (95\% CI: 9.4-48.0 months), which was considered to be satisfactory, as it was longer by 2.6 months compared with the PF regimen. Of note, this rather good median survival was obtained while M1a-stage patients were included in this study, contrary to the RTOG 94-05 study.

It is considered that this survival benefit may be acquired by using an accelerated radiation scheme. In China, Shi et al (15) initiated a study on LCAHRT for esophageal SCC treatment and yielded very encouraging results. Compared with conventional fractionation, the 5-year survival (34 vs. 15\%) and local control (55 vs. $21 \%$ ) rates were markedly improved with the LCAHRT regimen. Recently, three independent meta-analyses added to the evidence of LCAHRT being therapeutically beneficial for esophageal carcinoma (16-18). However, the optimal combination of chemotherapy regimens and accelerated radiation to maximize long-term survival remains to be determined. Zhao et al (4) reported the results of a phase III clinical trial on LCAHFR combined with PF, and the 1, 3 and 5-year survival rates were 67,44 and $40 \%$, respectively, in the combination group, and 77, 39 and 28\%, respectively, in the radiotherapy alone group $(\mathrm{P}=0.310)$; in addition, the incidence of grade $\geq 3$ toxicities were 42 and $25 \%$, respectively $(\mathrm{P}=0.05)$. Liu et al $(17)$ reported a meta-analysis on LCAHRT in esophageal carcinoma, including 21 randomized controlled trials, and the results indicated that LCAHRT combined with the PF regimen may improve the 5-year overall survival and 3-year local control in esophageal cancer compared with LCAHRT alone, with a significantly increased incidence of acute toxicities.

Pemetrexed was recently approved in combination with cisplatin as first-line treatment for advanced non-squamous-cell lung cancer and pleural mesothelioma. Pemetrexed combined with platinum compounds was also recommended for locally advanced head and neck SCC as an induction regimen $(19,20)$. However, there are very few data in the literature focusing on the treatment of esophageal SCC. To date, only one phase I study by Li et al (7) was conducted to evaluate the efficacy and safety of pemetrexed combined with cisplatin for locally advanced esophageal SCC. That study included 12 patients with T3-4N0-1M0-1a thoracic esophageal SCC. The total radiation dose administered was 59.6 Gy in $34 \mathrm{fx}$ in 5.4 weeks, and concurrent chemotherapy regimens were prescribed with cisplatin $10 \mathrm{mg} / \mathrm{m}^{2}$ on days $1-5$ and pemetrexed $400-500 \mathrm{mg} / \mathrm{m}^{2}$ once every 21 days. The tumor response was as high as $100 \%$, with CR in $66.7 \%$ (8/12) and PR in 
$33.3 \%$ (4/12) of the patients. Furthermore, no patient experienced cancer progression, with a median follow-up of 9 months (range, 3-22 months). Another phase II study investigated neoadjuvant pemetrexed/carboplatin combined with concomitant radiation for locally advanced esophageal cancer and gastroesophageal junction tumors (10). That phase II trial demonstrated antineoplastic activity, but did not achieve a complete pathological response (pCR). According to the results reported by Berger et al (21), overall survival was correlated with $\mathrm{pCR}$ and the 5-year survival of patients who achieved pCR following preoperative chemoradiotherapy was $\sim 50 \%$.

In the present study, although supportive treatment with oral folic acid and intramuscular vitamin B12 was routinely administered, the incidence of leukopenia and thrombocytopenia was higher with pemetrexed at a dose of $500 \mathrm{mg} / \mathrm{m}^{2}$ on day 1 once every 21 days, compared with that with $5-\mathrm{FU}$ (Table III). Generally, these toxicities were tolerable; in only 3 patients the consolidation chemotherapy was delayed due to grade 4 hematological toxicity (2 patients developed thrombocytopenia and 1 developed leukopenia), and only 2 deaths were considered possibly related to this treatment regimen (1 patient developed upper gastrointestinal hemorrhage and 1 patient developed esophagotracheal fistula). As regards overall survival, the present study demonstrated that the PP regimen was marginally superior to the PF regimen for locally advanced esophageal SCC (5-year survival rate, 40.9 vs. 33.2\%, respectively), although more patients with early-stage disease (IIa+IIb) were included in the PP group compared with the PF group (29.0 vs. $13.8 \%$, respectively). As demonstrated in Fig. 2, a trend toward better survival among patients who received the PP regimen was observed, but the difference did not reach statistical significance for this limited patient population.

Several strengths and limitations should be noted. This was only a retrospective study with a relatively small sample size, which may limit the generalizability of our findings. This study cohort consisted of an inhomogeneous patient population including patients with stage II, III and IVa disease, who had different prognoses following treatment.

In conclusion, the present study demonstrated that chemoradiotherapy with pemetrexed/cisplatin was similar with cisplatin/5-FU; however, the incidence of hematological toxicity was higher, whereas that of esophagitis was lower. These results should be validated in a large prospective cohort of patients.

\section{References}

1. Cooper JS, Guo MD, Herskovic A, Macdonald JS, Martenson JA Jr, Al-Sarraf M, Byhardt R, Russell AH, Beitler JJ, Spencer S, et al: Chemoradiotherapy of locally advanced esophageal cancer: Long-term follow-up of a prospective randomized trial (RTOG 85-01). Radiation Therapy Oncology Group. JAMA 281: 1623-1627, 1999

2. Minsky BD, Pajak TF, Ginsberg RJ, Pisansky TM, Martenson J, Komaki R, Okawara G, Rosenthal SA and Kelsen DP: INT 0123 (Radiation Therapy Oncology Group 94-05) phase III trial of combined-modality therapy for esophageal cancer: High-dose versus standard-dose radiation therapy. J Clin Oncol 20: $1167-1174,2002$

3. Kato K, Muro K, Minashi K, Ohtsu A, Ishikura S, Boku N, Takiuchi H, Komatsu Y, Miyata Y and Fukuda H; Gastrointestinal Oncology Study Group of the Japan Clinical Oncology Group (JCOG): Phase II study of chemoradiotherapy with 5-fluorouracil and cisplatin for Stage II-III esophageal squamous cell carcinoma: JCOG trial (JCOG 9906). Int J Radiat Oncol Biol Phys 81: 684-690, 2011.
4. Zhao KL, Shi XH, Jiang GL, Yao WQ, Guo XM, Wu GD and Zhu LX: Late course accelerated hyperfractionated radiotherapy plus concurrent chemotherapy for squamous cell carcinoma of the esophagus: A phase III randomized study. Int J Radiat Oncol Biol Phys 62: 1014-1020, 2005.

5. Higuchi K, Koizumi W, Tanabe S, Sasaki T, Katada C, Ishiyama $\mathrm{H}$ and Hayakawa $\mathrm{K}$ : A phase I trial of definitive chemoradiotherapy with docetaxel, cisplatin, and 5-fluorouracil (DCF-R) for advanced esophageal carcinoma: Kitasato digestive disease \& oncology group trial (KDOG 0501). Radiother Oncol 87: 398-404, 2008.

6. Chiarion-Sileni V, Corti L, Ruol A, Innocente R, Boso C, Del Bianco P, Pigozzo J, Mazzarotto R, Tomassi O and Ancona E: Phase II trial of docetaxel, cisplatin and fluorouracil followed by carboplatin and radiotherapy in locally advanced oesophageal cancer. Br J Cancer 96: 432-438, 2007.

7. Li BS, Gong HY, Huang W, Yi Y, Zhang ZC, Li HS, Wang ZT and Yu JM: Phase I study of concurrent selective lymph node late course accelerated hyper-fractionated radiotherapy and pemetrexed, cisplatin for locally advanced esophageal squamous cell carcinoma. Dis Esophagus 24: 251-257, 2011.

8. Seiwert TY, Connell PP, Mauer AM, Hoffman PC, George CM, Szeto L, Salgia R, Posther KE, Nguyen B, Haraf DJ and Vokes EE: A phase I study of pemetrexed, carboplatin, and concurrent radiotherapy in patients with locally advanced or metastatic non-small cell lung or esophageal cancer. Clin Cancer Res 13: 515-522, 2007.

9. Adjei AA: Pemetrexed (Alimta): A novel multitargeted antifolate agent. Expert Rev Anticancer Ther 3: 145-156, 2003.

10. Jatoi A, Soori G, Foster NR, Hiatt BK, Knost JA, Fitch TR, Callister MD, Nichols FC III, Husted TM and Alberts SR: Phase II study of preoperative pemetrexed, carboplatin, and radiation followed by surgery for locally advanced esophageal cancer and gastroesophageal junction tumors. J Thorac Oncol 5: 1994-1998, 2010.

11. Green FL, Page DL, Fleming ID, Fritzet AG, Balch CM, Haller DG and Morrow M: AJCC cancer staging manual, 6th edition. Ann Oncol 14: 345-346, 2003.

12. Li BS, Zhou T, Wang ZT, Li HS, Sun HF, Zhang ZC, Lin HQ, Wei YM, Gong HY, Huang W, et al: Phase I study of concurrent selective lymph node late course accelerated hyper-fractionated radiotherapy and Capecitabine, Cisplatin for locally advanced esophageal squamous cell carcinoma. Radiother Oncol 93: 458-461, 2009.

13. Therasse P, Eisenhauer EA and Verweij J: RECIST revisited: A review of validation studies on tumour assessment. Eur J Cancer 42: 1031-1039, 2006.

14. Trotti A, Colevas AD, Setser A, Rusch V, Jaques D, Budach V, Langer C, Murphy B, Cumberlin R, Coleman CN and Rubin P: CTCAE v3.0: Development of a comprehensive grading system for the adverse effects of cancer treatment. Semin Radiat Oncol 13: 176-181, 2003.

15. Shi XH, Yao W and Liu T: Late course accelerated fractionation in radiotherapy of esophageal carcinoma. Radiother Oncol 51: 21-26, 1999.

16. Zhang YW, Chen L, Bai Y and Zheng X: Long-term outcomes of late course accelerated hyper-fractionated radiotherapy for localized esophageal carcinoma in Mainland China: A meta-analysis. Dis Esophagus 24: 495-501, 2011.

17. Liu CX, Li XY and Gao XS: Meta-analysis of late course accelerated hyperfractionated radiotherapy combined with FP chemotherapy for esophageal carcinoma. Chin J Cancer 29: 889-899, 2010

18. Zhou ZG, Gao XS, Qiao XY and Zhang P: Literature analysis of radiotherapy for esophageal cancer in China. Chin J Cancer 29: 873-881, 2010.

19. Gilbert J, Murphy B, Dietrich MS, Henry E, Jordan R, Counsell A, Wirth P, Yarbrough WG, Slebos RJ and Chung CH: Phase 2 trial of oxaliplatin and pemetrexed as an induction regimen in locally advanced head and neck cancer. Cancer 118: 1007-1013, 2012.

20. Villaflor VM, Haraf D, Salama JK, Kocherginsky M, Langerman A, Gomez-Abuin G, Beniwal P, Blair EA, Stenson KM, Portugal L, et al: Phase II trial of pemetrexed-based induction chemotherapy followed by concomitant chemoradiotherapy in previously irradiated patients with squamous cell carcinoma of the head and neck. Ann Oncol 22: 2501-2507, 2011.

21. Berger AC, Farma J, Scott WJ, Freedman G, Weiner L, Cheng JD, Wang $\mathrm{H}$ and Goldberg $\mathrm{M}$ : Complete response to neoadjuvant chemoradiotherapy in esophageal carcinoma is associated with significantly improved survival. J Clin Oncol 23: 4330-4337, 2005. 